\title{
Influence of the Dry Friction Suspension System Characteristics on the Stick-Slip of Vertical Vibration of a Three-Piece Bogie
}

\author{
Dali Lyu $\mathbb{D}^{1},{ }^{1,2,3}$ Qichang Zhang $\mathbb{D}^{1,2}$ Kewei Lyu, ${ }^{4}$ Jiaxing Liu, ${ }^{1,2}$ and Yulong Li ${ }^{1,2}$ \\ ${ }^{1}$ Department of Mechanics, School of Mechanical Engineering, Tianjin University, Tianjin, China \\ ${ }^{2}$ Tianjin Key Laboratory of Nonlinear Dynamics and Control, Tianjin University, Tianjin, China \\ ${ }^{3}$ Tianjin Internal Combustion Engine Research Institute, Tianjin University, Tianjin, China \\ ${ }^{4}$ Technical Center, QiQiHar Railway Rolling Stock Co., Ltd, Qiqihar, China
}

Correspondence should be addressed to Qichang Zhang; qzhang@tju.edu.cn

Received 2 September 2020; Revised 28 October 2020; Accepted 9 January 2021; Published 29 January 2021

Academic Editor: Marcos Silveira

Copyright $(2021$ Dali Lyu et al. This is an open access article distributed under the Creative Commons Attribution License, which permits unrestricted use, distribution, and reproduction in any medium, provided the original work is properly cited.

\begin{abstract}
The dry friction structure is a commonly used vibration-damping method for railway vehicles. Insufficient vibration damping performance will cause excessive vibration of the vehicle, which is not conducive to the safety of the vehicle. However, the mechanism of vibration damping and the cause of clamping stagnation have not been well resolved. This paper uses the analytical method, numerical method, and finite element method to analyze the vertical dynamic characteristics of the simple suspension system with dry friction and demonstrates that the numerical method is an effective method to study the dry model. The conditions for the system to produce sticking events were analyzed by the numerical method. The analysis shows that the system's excitation is too small, which causes clamping stagnation to the system. The reduction of the wedge angle and the friction coefficient are conducive to eliminating sticking. A negative side frame angle is conducive to reducing the high-frequency energy of the excitation. Decreasing spring stiffness or increasing system mass to reduce system frequency can reduce sticking events. The mutual verification of different methods confirms the correctness of the analysis method and analyzes the cause of sticking or clamping stagnation from the mechanism, which provides a new idea for the design and improvement of the dry friction damping system of railway vehicle bogies.
\end{abstract}

\section{Introduction}

The three-piece bogies were first developed by the United States and the Soviet Union in the 1930s [1], comprised of one bolster, two side frames, and two wheelsets. As shown in Figure 1, the bolster is seated in the side frames through a set of springs and wedge friction dampers. The side frames are connected to wheelsets via bearing adapter. Now the threepiece bogies are the most common suspension for freight wagons across the world for its low manufacturing cost and low maintenance cost.

The design of robust limits the critical speed of the empty wagon for low cost in production [2]. The bogies play key roles in promoting the running behavior of rail vehicles. The vibration energy from track disturbances translates to wagons via the wheelsets, side frames, and the bolster. It dissipates primarily through dry friction damper. The friction wedges in three-piece bogies play a controlling role in the vertical and lateral dynamics, as well as the lateral stability of wagons. Owing to increasing demands for high load capacity and high-speed rail vehicles, stability and safety have been investigated using diverse methods.

The characteristic of dry friction plays a significant role in the dynamic system. The modeling of the friction force in the system depends on several environmental factors including sliding speed, temperature, and normal load [3]. The magnitude of the friction force decreases when the relative velocity $|\dot{x}|$ of the contact surface of two solids increases if $|\dot{x}|$ 


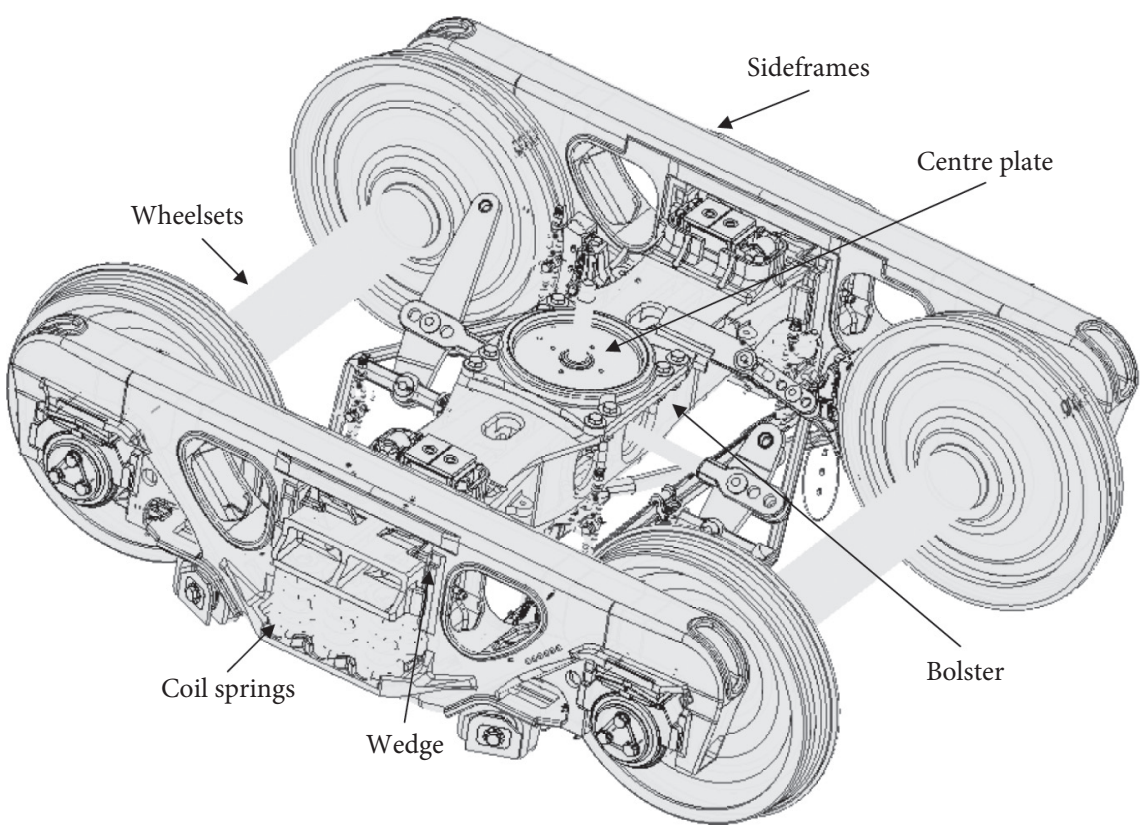

Figure 1: Bogie structure.

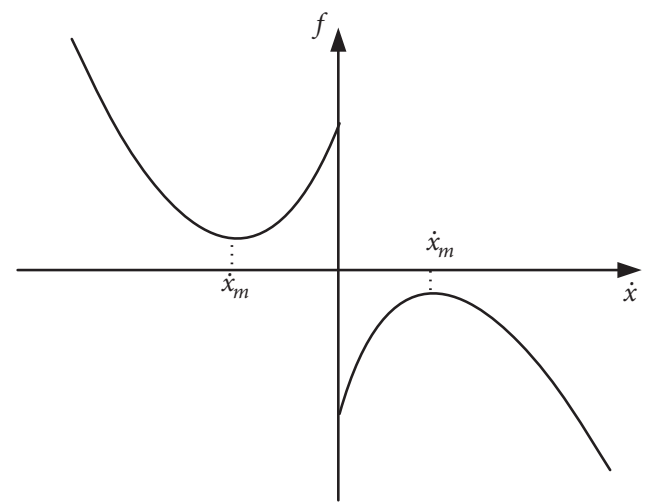

FIGURE 2: Friction force as a function of velocity.

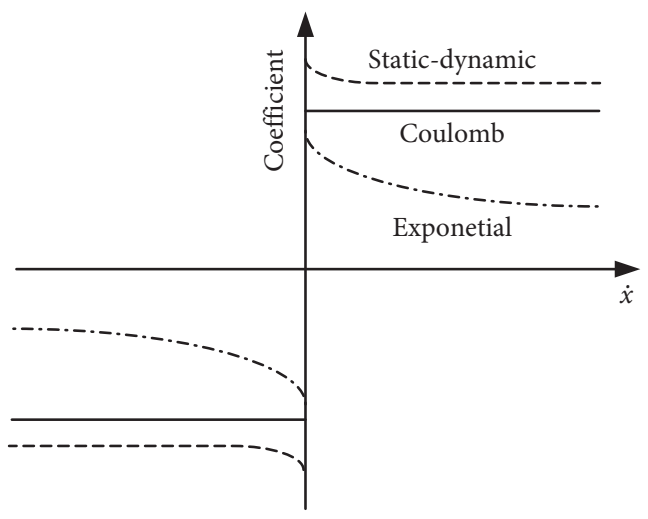

FIgURE 3: Friction models.

is smaller than the critical value $\left|\dot{x}_{m}\right|$, and then it increases when $|\dot{x}|$ becomes larger than $\left|\dot{x}_{m}\right|$ [4] as shown schematically in Figure 2. As shown in Figure 3, Coulomb friction model, static and dynamic friction model, and exponential friction model are the most commonly used friction models in wedge suspension simulation [5]. The static-dynamic friction model, that is, different coefficients between the static friction and the kinetic friction, was used to study 
bifurcations [6]. The relative velocity dependent friction model that includes the nonlinear function of the relative sliding velocity was used to research the corresponding stick-slip motion for the spring-mass model. The qualitative behavior was equal for both the static-dynamic friction model and the relative velocity dependent friction model [7] and was equal for the Coulomb friction model and the staticdynamic friction model [8].

The modeling of the friction force was simply described as a combination of springs and dampers, and applications have been seen of springs and friction elements in parallel and in series, which are normally developed in commercial simulation programs, such as NUCARS, VAMPIRE, ADAMS, and multibody system (MBS) simulation package. Sperry developed a bogie model with friction wedge components, in which the friction characteristics of the wedge are replaced by the established contact point, and each contact face of the friction wedges had twenty-five contact points, setting up in a five-by-five grid [9]. Li [10] developed the model with 6 DOF rigid wedges body, bolster and damper spring parameters setting, and four contact areas by SIMPACK software. The model reflected the wedge and the bolster actual motion. The vibration damping characteristics of the wedges under actual motion conditions were analyzed, but there was a lack of analysis of the stick-slip phenomenon and the stagnation of wedges.

A data-driven model of dynamics of rail vehicle was formulated to replace the original mechanical element by machine learning. The method assumes a set of sample data with the corresponding set of observed outputs and predicts the underlying relationship between the inputs and the outputs [11]. A Kriging surrogate model of Y25 bogies was constructed to reflect the relationship between the wheel flats, vehicle speeds, and axle box acceleration. The results of simulation and experimental demonstrated that the method can estimate the length of wheel-flats [12]. The stochastic models of single suspension and lateral suspension were trained, which can represent the behavior of the suspension system and investigate the behavior of a multibody dynamics model by machine learning from computer simulations [13]. This method uses machine learning techniques to extract nonlinear features from known data and replaces the original mechanical elements with a surrogate element. However, it does not need to analyze the mechanism of nonlinearity, and it is also unable to analyze the stick-slip phenomenon of the wedge damping model.

Xia [14] modeled suspension considering nonlinear continuous dry friction using the static and kinetic dry friction coefficients. A new conception of friction direction angles is proposed [15] in the cases of stick state and slip state. This model can calculate the friction force vector in the cases of one-dimensional dry friction and two-dimensional dry friction. Zhang [16] investigated the lateral dynamics of the suspension system with linear springs and dry friction dampers, indicating that period motions, quasiperiodic motions, and chaotic motions are the main dynamic characteristics of the suspension system. Baruffaldi [17] described a model using a linear complementarity approach for friction dampers, showing that friction coefficient can affect the general damping of the dynamic response and the development of nonlinear phenomena related to stick-slip. Sun analyzed the wedge friction force, the bolster displacement, and the tangent and normal forces on wedge angular surface during suspension bounce modes [18] and pitch modes [19] using a two-dimensional finite element method (FEM). In the railway wagon industry, the special line dynamics test was carried out to study the impact of the secondary suspension system of the $\mathrm{C}_{80}$ type aluminum alloy car on the dynamic performance in the railway wagon industry. The analysis obtained that the increase of the dynamic friction coefficient increased the vertical vibration acceleration of the car body, and the second series stiffness decreased to reduce vertical acceleration [20].

In these references, some researches have been done on the influence of friction on the dynamic characteristics of the system, but the rationality of the analysis method has not been confirmed. Experimental methods have been used to study the influence of simple structure or suspension parameters of railway wagons on dynamics. Some methods only conduct a qualitative analysis of the structure but do not dig for deeper reasons, or the model studied is very different from the actual structure and cannot guide the structural design of the vibration reduction system. Therefore, this paper first analyzes the rationality of the method for studying dry friction and confirms that it is suitable for the analysis method of dry friction system with stick-slip motion. Then, the causes of sticky events and methods to reduce sticky events are determined by parameter analysis. The purpose of this article is to analyze the causes of stick-slip in wedge suspensions and explore ways to reduce this phenomenon.

\section{Mechanical Model of the Suspension Model}

According to the results of the special line dynamics test, it can be found that the vertical floating motion accounts for $80 \%$ of the total vehicle operation [21], so the study of vertical vibration characteristics is first carried out. The research model of this paper is the model established in reference [22]. The physical components and typical schematic representation of half of the secondary suspension model are shown in Figure 4. The specification of the simple model is described in Table 1. The primary function of the wedge is to provide damping in the vibration motion, and all of the elements are assumed to be rigid. In this model, the variables $X$ and $Y$ indicate horizontal and vertical displacements, respectively. The subscripts $w, b$ and $s$ indicate wedge, bolster, and side frame, respectively. The displacement of the side frame $Y_{s}$ refers to $X_{w}$ the inertial frame at the ground, the horizontal displacement of the wedge $X_{w}$ and the bolster $X_{b}$ refer to the centerline of the bolster, and the vertical displacement of both the wedge $Y_{0}$ and the bolster $Y_{b}$ refer to the position of side frame. The initial precompression of wedge and bolster springs is denoted by $Y_{w 0}$ and $Y_{b 0}$, respectively. The contact forces are denoted by $F$ for friction and $N$ for normal forces, the spring stiffness is denoted by, and the mass is denoted by $K$. The friction 


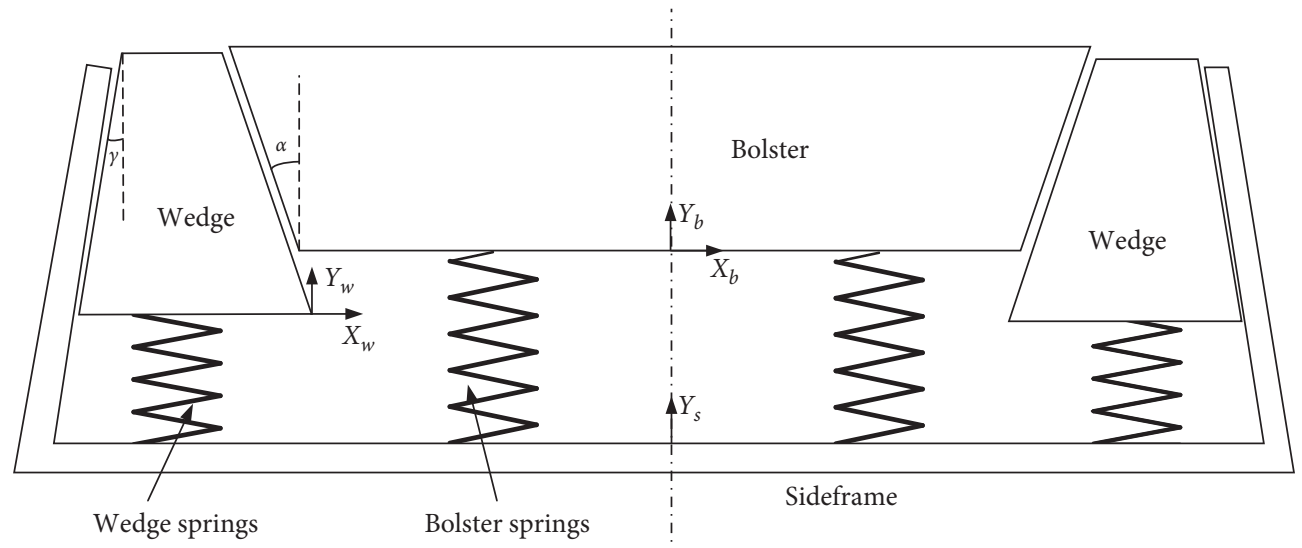

Figure 4: Variable damping suspension.

TABLE 1: Specification of the suspension model.

\begin{tabular}{lc}
\hline Parameter & Specification \\
\hline Mass of each wedge & $0.17 \mathrm{~kg}$ \\
Mass of bolster & $0.7 \mathrm{~kg}$ \\
Effective stiffness of each wedge group of springs & $2550.6 \mathrm{~N} / \mathrm{m}$ \\
Effective stiffness of each bolster group of springs & $5101.2 \mathrm{~N} / \mathrm{m}$ \\
Wedge angle & $37.5^{\circ}$ \\
Side frame angle & $4^{\circ}$ \\
Friction coefficient at all sliding interfaces & 0.29 \\
Offset of wedge springs (precompression) & $-3.46 \mathrm{~mm}$ \\
Offset of bolster springs (pretension) & $3.04 \mathrm{~mm}$ \\
\hline
\end{tabular}

factors of interfaces are wedge-side frame $\mu_{w s}$ and wedgebolster $\mu_{w s}$. The sign convention for the forces corresponds to the given configuration, where the wedge and bolster units are displaced above their equilibrium positions.
Due to the symmetry of the structure, the horizontal displacement of the bolster $X_{b}$ equals zero in the vertical vibration system. All contacts of wedge, side frame, and bolster are assumed constant. The remaining three configuration variables should satisfy the following sliding constraints:

$$
\begin{array}{r}
X_{w}=\tan (\gamma) Y_{w} \\
\tan (\alpha) Y_{w}+X_{w}=\tan (\alpha) Y_{b}
\end{array}
$$

As shown in Figure 5, the wedge is loaded differently during loading and unloading situations of suspension movements. The direction of the friction force is opposite to the speed. According to D'Alembert's principle, the dynamic equations of the wedge are

$$
\begin{aligned}
\sum F_{x} & =0-F_{w s} \sin \gamma+F_{n w s} \cos \gamma+\left(-F_{w b}\right) \sin \alpha-F_{n w b} \cos \alpha-M_{w} \ddot{X}_{w}=0 \\
\sum F_{y} & =0 \quad-F_{n w s} \sin \gamma-F_{w s} \cos \gamma+\left(-K_{w}\left(Y_{w}+Y_{w 0}\right)\right)-M_{w}\left(\ddot{Y}_{w}+\ddot{Y}_{s}+g\right)-F_{n w b} \sin \alpha-\left(-F_{w b}\right) \cos \alpha=0 \\
F_{w s} & =\mu F_{n w s} \\
F_{w b} & =\mu F_{n w b} .
\end{aligned}
$$

This can easily be solved to get the normal force:

$$
F_{n w b}=\frac{\left(K_{w}\left(Y_{w}+Y_{w 0}\right)+M_{w}\left(\ddot{Y}_{w}+g-\ddot{Y}_{s}-R_{w s} \tan \gamma \ddot{Y}_{w}\right)\right)}{R_{w b}},
$$

$$
\begin{aligned}
R_{w s} & =\frac{\mu_{w s} \operatorname{sgn}\left(\dot{Y}_{w}\right) \cos \gamma-\sin \gamma}{\mu_{w s} \operatorname{sgn}\left(\dot{Y}_{w}\right) \sin \gamma+\cos \gamma}, \\
R_{w b} & =\left(R_{w s} \sin \alpha-\cos \alpha\right) \mu_{w b} \operatorname{sg}\left(\dot{Y}_{w}\right)+\sin \alpha+R_{w s} \cos \alpha .
\end{aligned}
$$

where $R_{w s}$ and $R_{w b}$ are functions of both the suspension parameter values and the friction model, as given by
As shown in Figure 6, when the bolster is loaded during loading, the dynamic equation of half bolster is

$$
\sum F_{y}=0 \quad F_{n w b} \sin \alpha-F_{w b} \cos \alpha-\frac{K_{b}}{2}\left(Y_{b}+Y_{b 0}\right)-\frac{M_{b}}{2}\left(g+\ddot{Y}_{b}+\ddot{Y}_{s}\right)=0 .
$$




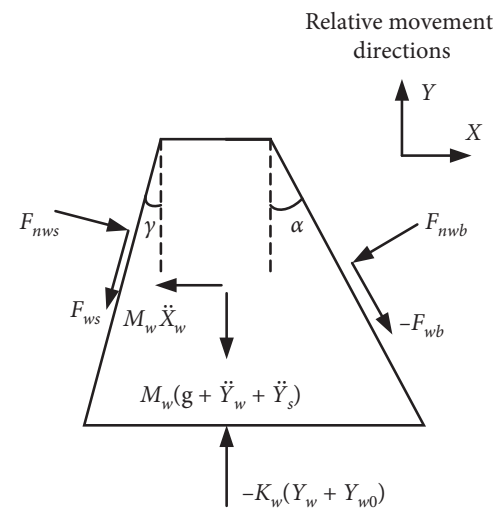

FIGURE 5: Mechanical model of the wedge.

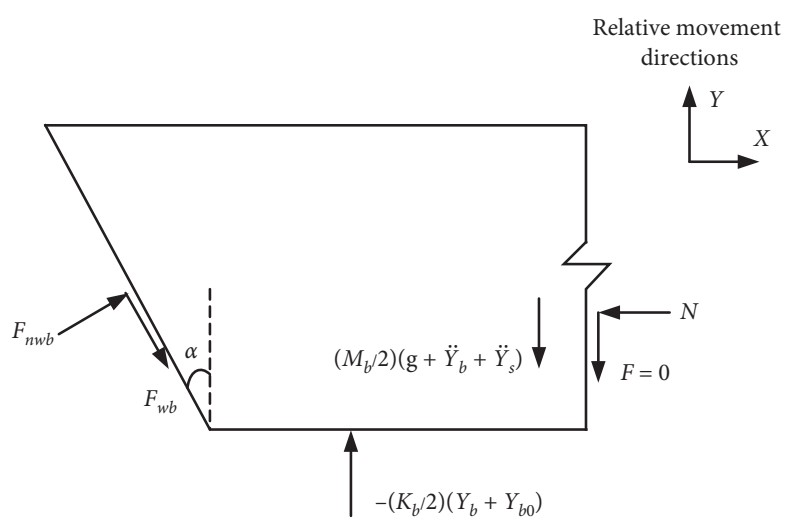

Figure 6: Mechanical model of the bolster.

Upon substituting Equation (3) into Equation (5), we can get

$$
\ddot{Y}_{b}+\frac{2 K_{w} \Gamma R_{\alpha \gamma}+K_{b}}{2 R_{\alpha \gamma} \Gamma M_{w}\left(1+\tan \gamma R_{w s}\right)+M_{b}} Y_{b}+\frac{2 M_{w} R_{\alpha \gamma}+M_{b}}{2 R_{\alpha \gamma} \Gamma M_{w}\left(1+\tan \gamma R_{w s}\right)+M_{b}}\left(\ddot{Y}_{s}+g\right)+\frac{2 K_{w} Y_{w 0} R_{\alpha \gamma}+K_{b} Y_{b 0}}{2 R_{\alpha \gamma} \Gamma M_{w}\left(1+\tan \gamma R_{w s}\right)+M_{b}}=0,
$$

where $\Gamma$ and $R_{\alpha \gamma}$ are functions of both the suspension parameter values and the friction model, as given by

$$
\begin{aligned}
\Gamma & =\frac{\tan \alpha}{\tan \alpha+\tan \gamma}, \\
R_{\alpha \gamma} & =\frac{\sin \alpha-\mu_{w b} \operatorname{sgn}\left(\dot{Y}_{w}\right) \cos \alpha}{R_{w b}} .
\end{aligned}
$$

The equation is transformed into a nondimensional form by parameter scaling and coordinate transformations.

The side frame displacement $Y_{s}$ is assumed to be a cosine periodic disturbance, as given by

$$
Y_{s}=A \cos (\omega t)
$$

where $A$ is the displacement amplitude of the disturbance and $\omega$ is the frequency.

The natural frequency of the bolster element is defined by $\omega_{0}$ :

$$
\omega_{0}=\sqrt{\frac{K_{b}}{M_{b}}}
$$

The rescale time (i.e., t) is converted to the nondimensional time $\tau$ :

$$
\tau=\omega_{0} t .
$$

The differentiations with respect to the time variable $t$ are then rescaled as 


$$
\begin{gathered}
\frac{d}{\mathrm{~d} t}=\omega_{0} \frac{d}{\mathrm{~d} \tau}, \\
\frac{d^{2}}{\mathrm{~d} t^{2}}=\omega_{0}^{2} \frac{d^{2}}{\mathrm{~d} \tau^{2}} .
\end{gathered}
$$

Using the rescaling, equations (6) and (8) can be combined into a nondimensional form as

$$
\ddot{y}+f y+h+l\left(g-a \psi^{2} \cos (\psi \tau)\right)=0,
$$

where $a=\left(\omega_{0}^{2} / g\right) A, \psi=\omega / \omega_{0}, y=\left(\omega_{0}^{2} / g\right) Y_{b}$ and $f, h, l$ are functions of both the suspension parameter values and the friction model, as given by

$$
\begin{aligned}
& f=\frac{2 K_{w} \Gamma R_{\alpha \gamma}+K_{b}}{2 R_{\alpha \gamma} \Gamma M_{w}\left(1+\tan \gamma R_{w s}\right)+M_{b}}, \\
& h=\frac{2 K_{w} Y_{w 0} R_{\alpha \gamma}+K_{b} Y_{b 0}}{2 R_{\alpha \gamma} \Gamma M_{w}\left(1+\tan \gamma R_{w s}\right)+M_{b}}, \\
& l=\frac{2 M_{w} R_{\alpha \gamma}+M_{b}}{2 R_{\alpha \gamma} \Gamma M_{w}\left(1+\tan \gamma R_{w s}\right)+M_{b}} .
\end{aligned}
$$

\section{Solution Method}

3.1. Approximate Analytical Methods. The harmonic balance (HB) method is an efficient quantitative analysis method to solve both strongly and weakly nonlinear dynamic systems. The HB method has been made to improve the accuracy of calculation or simplify the calculation process. To obtain higher precision periodic solutions, the incremental harmonic balance (IHB) method was proposed [23]. Combining the $\mathrm{HB}$ method with the incremental harmonic balance approach, an improved incremental harmonic balance method was presented to obtain the higher-order approximate steady-state solutions for strongly nonlinear systems [24]. Wu [25] used the HB method for Yao-Cheng oscillator, showing that the $\mathrm{HB}$ method is as effective as the homotopy perturbation method for Yao-Cheng oscillator, and the $\mathrm{HB}$ method has the advantages of no need for construction and no expansion for the solution and the parameter. The approximate solution of the response of equation (12) is established by using the HB method.

Based on the HB method, the first term of the general Fourier series solution is used as the expression for the solution of the equation. The solution can be supposed as

$$
y(\tau)=y \cos (\psi \tau+\theta)
$$

where $y(\tau)$ denotes the first-order approximate solution, $y$ is the amplitude, $\theta$ is the initial phase angle, and $\psi$ is the nondimensional fundamental of the harmonic excitation.

Upon substituting equation (14) into the system equation (12), the following expression is obtained:

$$
-\psi^{2} y \cos (\theta+\tau \psi)+f\left(y^{\prime}(\tau)\right) y \cos (\theta+\tau \psi)+h\left(y^{\prime}(\tau)\right)+l\left(y^{\prime}(\tau)\right)\left(g-a \psi^{2} \cos (\tau \psi)\right)=0
$$

where the coefficient functions $f, h, l$ are corresponding variables to the relative velocity of the system motion.

Since the coefficient functions $f, h, l$ contain discontinuous dependence on the unknowns, there is no direct way to make every coefficient of the harmonics equal to zero. When the velocity direction changes, the coefficients of the dry friction system change. Under this situation, the system's mechanical properties are nonlinear, as well as the formation of an equivalent system of two smooth differential equations. The discontinuity at $\dot{y}(\tau)=0$ of the friction models produces a natural division of the phase space at the sticking plane $S=\{(y, \dot{y}, \tau) \mid \dot{y}=0\}$. Equation (15) can be written in two parts:

$$
\begin{array}{ll}
-\psi^{2} y \cos (\theta+\tau \psi)+f^{+} y \cos (\theta+\tau \psi)+h^{+}+l^{+}\left(g-a \psi^{2} \cos (\tau \psi)\right)=0, & S^{+}=\{(y, \dot{y}, \tau) \mid \dot{y} \geq 0\}, \\
-\psi^{2} y \cos (\theta+\tau \psi)+f^{-} y \cos (\theta+\tau \psi)+h^{-}+l^{-}\left(g-a \psi^{2} \cos (\tau \psi)\right)=0, & S^{-}=\{(y, \dot{y}, \tau) \mid \dot{y} \leq 0\},
\end{array}
$$

$$
\dot{y}(\tau)=-y \psi \cos (\psi \tau+\theta)
$$

where the coefficients $f, h, l$ are appended with superscripts $( \pm)$ to indicate different domains. Positive $(\operatorname{sgn}(\dot{y})=1)$ indicates that the system is moving up, and negative (sgn $(\dot{y})=-1)$ indicates the contrast.

In order to determine the direction of relative direction motion of the system, the derivative of equation (14) is carried out to obtain the relative speed of the system:
Let $\varphi=\psi \tau+\theta$. For $0 \leq \varphi \leq \pi, \operatorname{sgn}(\dot{y}(\tau) t<n 0)=-1$, the equation (16b) is multiplied by $\sin \varphi$ and $\cos \varphi$ respectively, the integration covers the complete period. For $\pi \leq \varphi \leq 2 \pi$, $\operatorname{sgn}(\dot{y}(\tau) t>n 0)=1$, the equation (16a) is multiplied by $\sin \varphi$ and $\cos \varphi$ respectively, and the integration covers the complete period. The following expression is obtained: 


$$
\begin{aligned}
& \pi a l^{+} \psi^{2} \sin (\theta)+\pi a l^{-} \psi^{2} \sin (\theta)+4\left(l^{+}+h^{+}\right) \\
& \quad-4\left(l^{-}+h^{-}\right)=0, \\
& y\left(f^{+}-\psi^{2}\right)+y\left(f^{-}-\psi^{2}\right)-a l^{+} \psi^{2} \cos (\theta) \\
& \quad-a l^{-} \psi^{2} \cos (\theta)=0 .
\end{aligned}
$$

The following response functions for the amplitude and phase coefficients are obtained:

$$
\begin{aligned}
& y=\sqrt{\frac{a^{2}\left(l^{+}+l^{-}\right)^{2} \pi^{2} \psi^{4}-16\left(l^{+}-l^{-}+h^{+}-h^{-}\right)^{2}}{\pi^{2}\left(f^{+}+f^{-}-2 \psi^{2}\right)^{2}}}, \\
& \theta=\operatorname{Arcsin}\left(\frac{4\left(l^{+}-l^{-}+h^{+}-h^{-}\right)}{a\left(l^{+}+l^{-}\right) \pi \psi^{2}}\right) .
\end{aligned}
$$

3.2. Numerical Implementation. The solution of the equation calculated by the first-order HB method can only represent the change of the system over the whole period but cannot analyze the stick-slip phenomenon in a period. Therefore, the numerical calculation method is adopted to analyze the movement of the system. The numerical integration of equations (16a) and (16b) is performed by a standard fourthorder Runge-Kutta method [26], with a maximum step size of 0.001 .

The nondimensional equation of motion is a nonsmooth differential equation. The phase flow defined by equation (12) alternates between upward motion and downward motion. The sticking region is founded by evaluating equation (12) where their respective phase flows are stationary $S_{0}$, that is, where $\ddot{y}=\dot{y}=0$,

$$
S_{0}=\left\{(y, \dot{y}=0) \mid y_{0}^{+} \leq y \leq y_{0}\right\}
$$

where $y_{0}^{+}$and $y_{0}^{-}$are the sticking region of upward and downward motion, respectively, as given by

$$
\begin{aligned}
& y_{0}^{+}=-\frac{h^{+}+l^{+}\left(g-a \psi^{2} \cos (\psi \tau)\right)}{f^{+}}, \\
& y_{0}^{-}=-\frac{h^{-}+l^{-}\left(g-a \psi^{2} \cos (\psi \tau)\right)}{f^{-}} .
\end{aligned}
$$

3.3. Finite Element Modeling of the Suspension System. The mechanical model of the motion system simplified structure details may not accurately describe the friction force characteristics during the movement of the bogie. The suspension 3D model is established by FEM, and the rationality of model simplification is judged by comparing the simulation results of the mechanical model and the FEM model. The suspension can be modeled using its quarter structure from a symmetric surface. The quarter suspension finite element model is shown in Figure 7. The three-dimensional model is established using ABAQUS. The bolster and wedge are suspended on the side frame by spring. The dry friction exists between the wedge and the bolster, as well as between the wedge and the side frame, and the friction coefficients at all sliding interfaces are equal to 0.29 .

The contact surfaces of the wedge, bolster, and side frame are defined as the contact pairs, and the friction characteristics will influence the response of the system motion. In this model, the penalty friction formulation in ABAQUS is selected for the contact calculation of the contact pairs. The longitudinal and lateral movements of the side frame and bolster at their symmetric surfaces are restrained, and the lateral movement of the wedge at symmetric surfaces is restrained. The model boundary conditions are shown in Figure 8 .

3.4. Methods of Comparison. The vibration isolation rate is an efficient index to evaluate the vibration isolation performance of the isolator, which can reflect the overall relative motion of the suspension system. The vibration isolation rate [27] is defined as the ratio of the response energy of the suspension system to the excitation energy of the base, expressed as

$$
T_{s}=20 \log \left(\frac{y_{(\tau) r m s}}{a \psi^{2} / \sqrt{2}}\right) .
$$

Upon substituting the physical parameter values in Table 1 into equation (13), the coefficients can be written as follows: for $S^{+}=\{(y, \dot{y}, \tau) \mid \dot{y} \geq 0\}, f^{+} \approx 1.1918, h^{+} \approx 1.0122$, $l^{+} \approx 0.7561 ; \quad$ for $\quad S^{-}=\{(y, \dot{y}, \tau) \mid \dot{y} \geq 0\}, \quad f^{-} \approx 1.3735$, $h^{-} \approx 1.0369, l^{-} \approx-0.5237$. The response functions can be written as

$$
\begin{aligned}
& y=\sqrt{\frac{-0.6384+1.0497 a^{2} \psi^{4}}{\left(-1.2827+\psi^{2}\right)^{2}}}, \\
& \theta=-\arcsin \left(\frac{0.7799}{a \psi^{2}}\right) .
\end{aligned}
$$

Figure 9 shows the vibration isolation rates obtained by the $\mathrm{HB}$ method and numerical method, respectively. The simulation results have very good consistency, indicating that both the analytical method and the numerical method are feasible for analyzing the vibration isolation rate. However, it is important to note that the first-order $\mathrm{HB}$ method relies on the assumption that the system response can be well approximated to a periodic function and may not be applicable to other systems. The values of the peaks and troughs of the time domain curve of the system obtained by the numerical method are constant, as shown in Figure 10(a), and the frequency domain curve of the system obtained by the numerical method has higher-order frequency in the system response as shown in Figure 10(b). The high-frequency response of the suspension system is very harmful to the wagon. The HB method only considers the first-order influence on the system. The $\mathrm{HB}$ method can evaluate the vibration isolation performance of the system, but it cannot obtain the detailed features of the system 


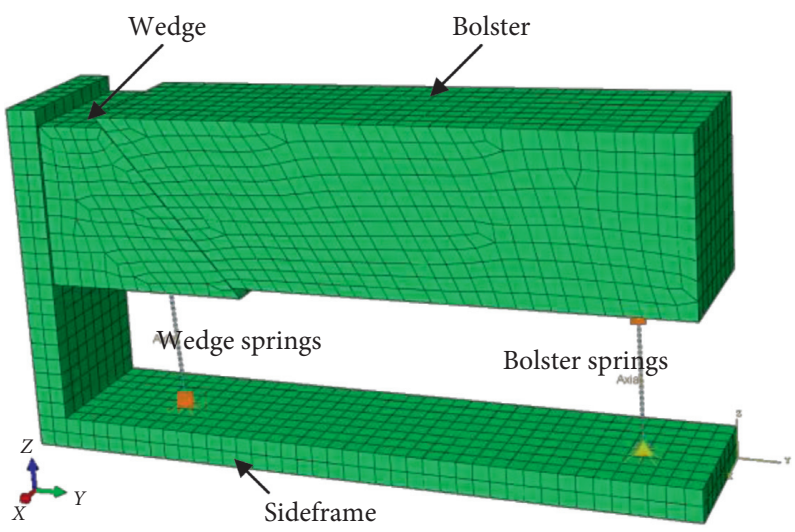

FIgURe 7: Quarter bogie model.

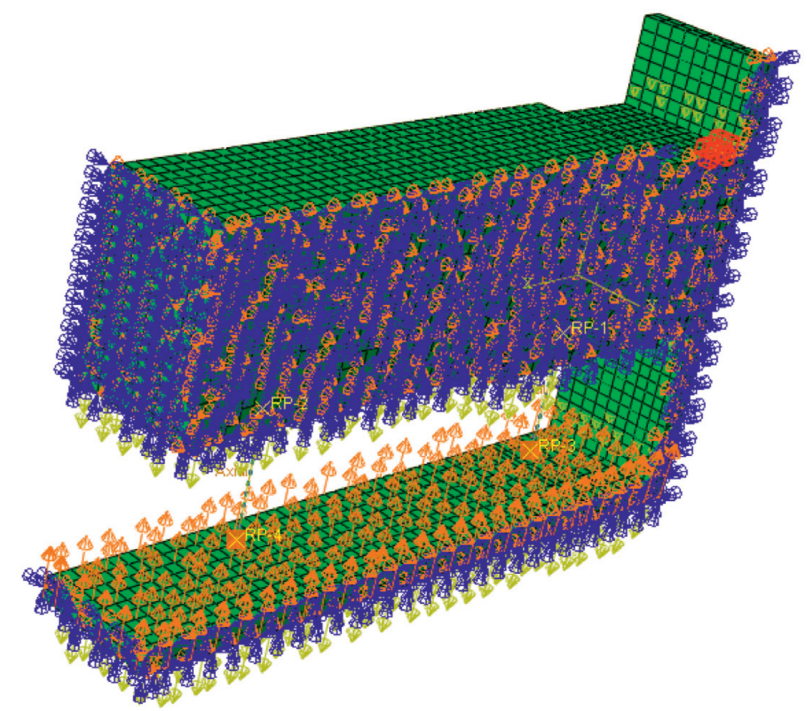

FIGURE 8: Model boundary condition.

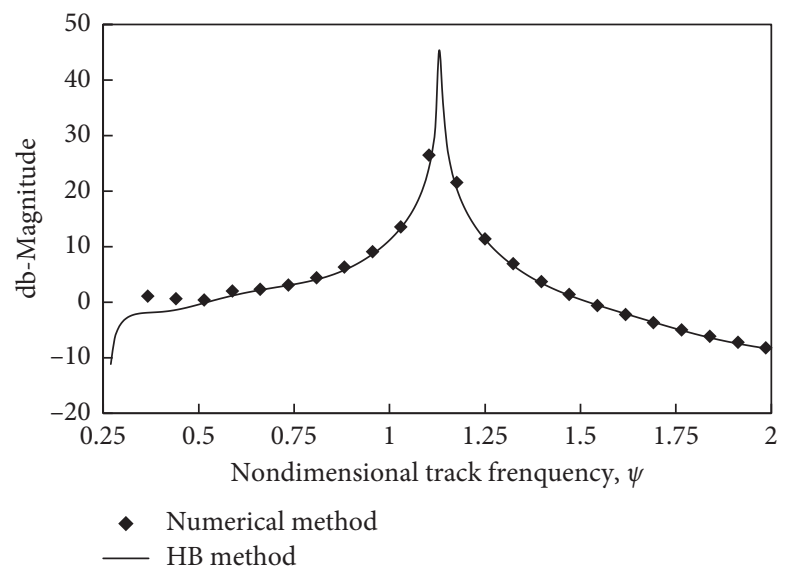

FIGURE 9: Results comparison when $a=11.14$ for $\mathrm{HB}$ and numerical method.

during a period. Therefore, the numerical method is more suitable for evaluating the dynamic characteristics of the suspension system.
The validity of the HB method is confirmed by numerical analysis, but the correctness of the established mechanical model cannot be proved. The FEM based on the structural 


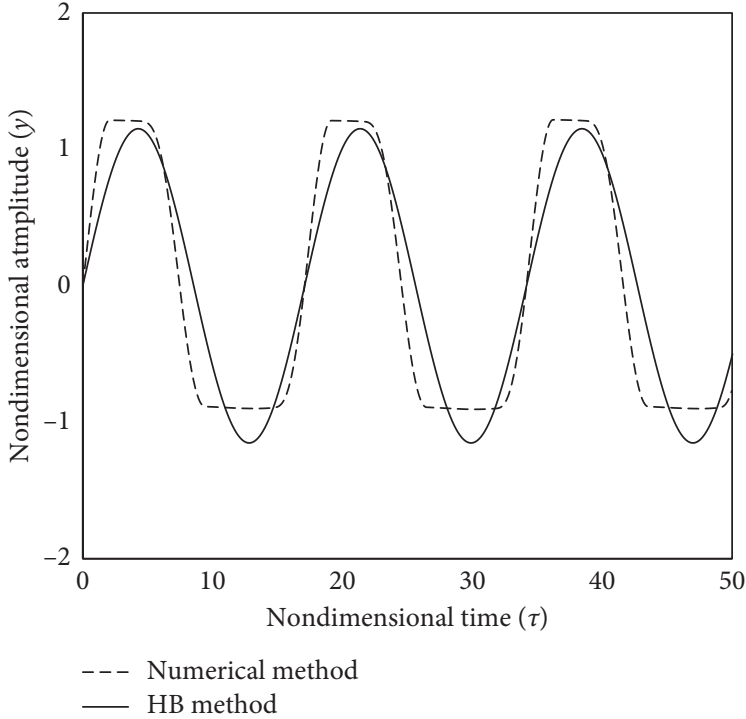

(a)

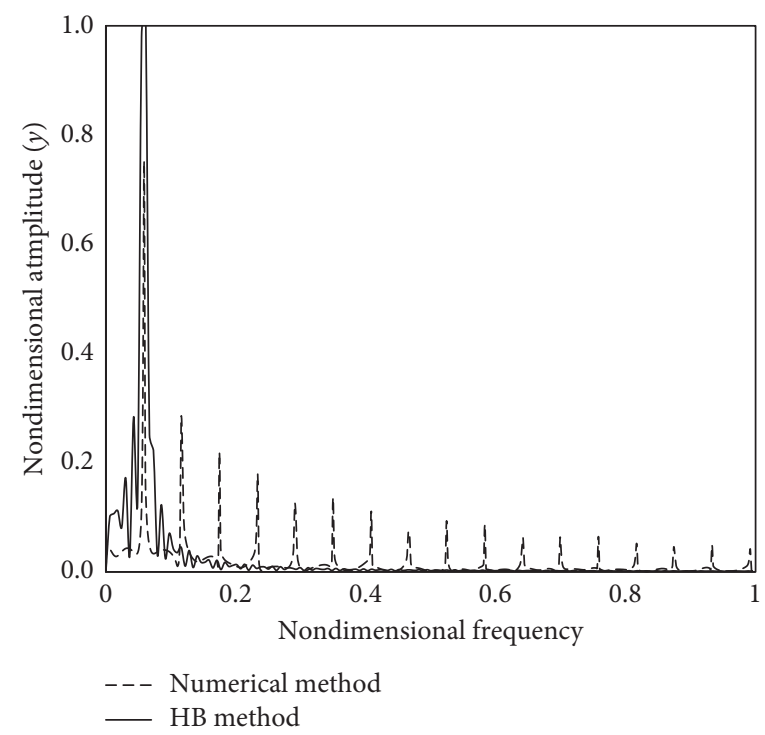

(b)

Figure 10: The results of HB and numerical method when $a=11.14, \psi=0.368$. (a) Time-domain curves. (b) Spectrum curves.

parameters of the model can be used to verify the rationality of the mechanical model. The system responses under excitation with the amplitude of $15 \mathrm{~mm}$ and frequency of $5 \mathrm{~Hz}$, $7 \mathrm{~Hz}$, and $10 \mathrm{~Hz}$ are calculated. Figure $11(\mathrm{a})$ is similar to the case of a sinusoidal waveform. Figure 11(b) shows a constant displacement at the trough, and Figure 11(c) shows a constant displacement at both the peak and the trough. It can be seen that the waveform and amplitude of the time domain curves are relatively close, which proves the correctness of the mechanical model establishment.

Comparing the results of the analysis method, the numerical method, and the FEM, the analytical method can reflect the overall response energy, but the details cannot be estimated. The FEM can consider all details of the system. However, the calculation of the time cost is highest. The numerical method can be used to assess overall energy and details in suspension system, in which the efficiency and accuracy are fitted for the requirements.

\section{Discussions}

4.1. The Effect of Excitation on the Response. The system responses with the excitation amplitude changing from small to large are calculated, respectively. The results are shown in Figure 12. It can be found that the system response increases as the excitation amplitude increases. Through the displacement response waveform, when the excitation amplitude is relatively small, the sticking phenomenon occurs at the peak and trough. When the excitation energy increases, the sticking events at the peak disappear firstly, and the sticking events at the trough disappear.

The system responses with the excitation frequency changing from $2 \mathrm{~Hz}$ to $10 \mathrm{~Hz}$ are calculated, respectively. The results are shown in Figure 13. When the excitation frequency is $2 \mathrm{~Hz}$, it is in a permanent sticking state. With the increase of the excitation frequency, the phenomenon of motion is the same as the excitation amplitude, which is consistent with the situation that railway wagons are prone to stagnation at low speeds [28]. The contact surface pressure is determined by the resultant force on the bolster. The friction forces of relative downward movement are greater than those of the upward movement so that the sticking events at the wave peak disappear earlier than the trough.

\subsection{The Effect of Different Structure Parameters on the Response}

4.2.1. The Different Stiffness and Mass of the Bolster. With the stiffness of the bolster spring increasing from $2500 \mathrm{~N} / \mathrm{m}$ to $6500 \mathrm{~N} / \mathrm{m}$, as shown in Figure 14, the relative displacement of bolster decreasing is observed to be constant, and the sticking events appear. The phenomena are similar to the change of mass of the bolster decreasing from $1.3 \mathrm{~kg}$ to $0.1 \mathrm{~kg}$, as shown in Figure 15 because the angular frequency is positively correlated with stiffness and negatively correlated with mass. The quality of railway wagons is very different between empty cars and loaded cars. The smaller stiffness of bolster spring and the larger mass of the bolster reduced the normal frequency of suspension system, and the lower frequency of the system is beneficial in eliminating sticking events and improving the vibration damping performance of the suspension system [20]. Generally, the amplitude of the vehicle vibration of the loaded cars is smaller than that of empty cars, but the vibration of the container flat was larger, and the spectrum analysis has more high-frequency energy [29], which may be related to the secondary suspension. The presence of sticking events causes high-frequency energy to be transferred to the vehicle body, as shown in Figure 11(b). 


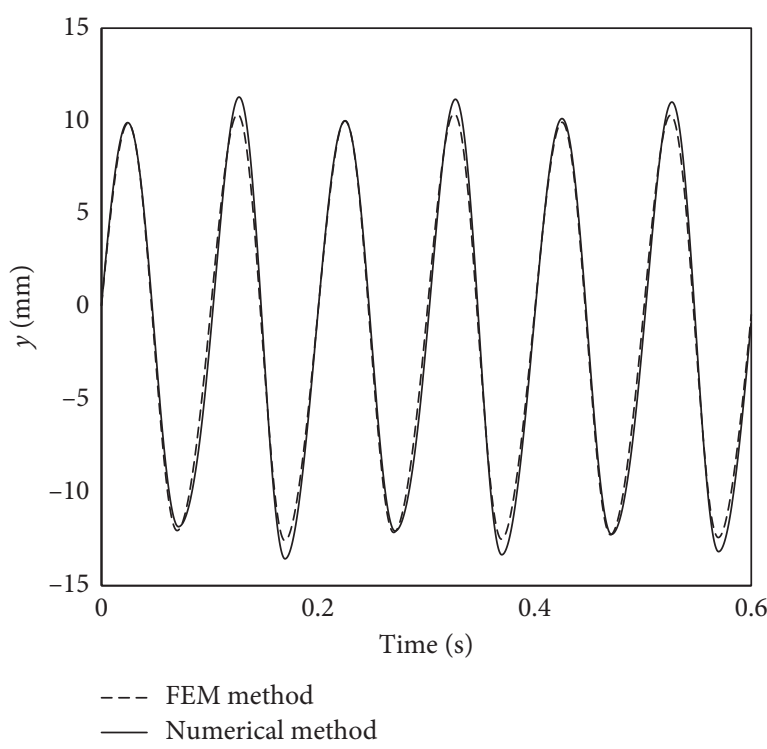

(a)

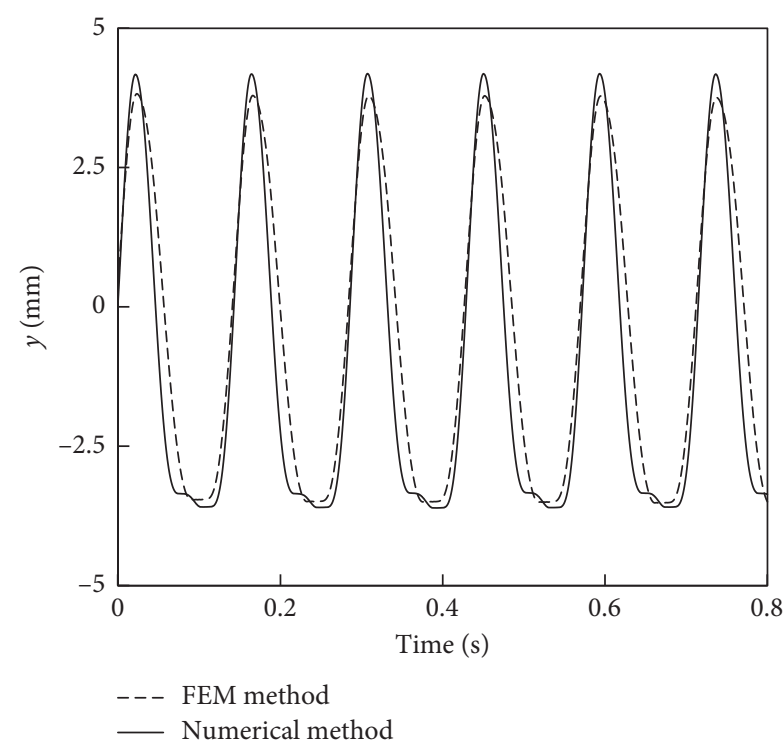

(b)

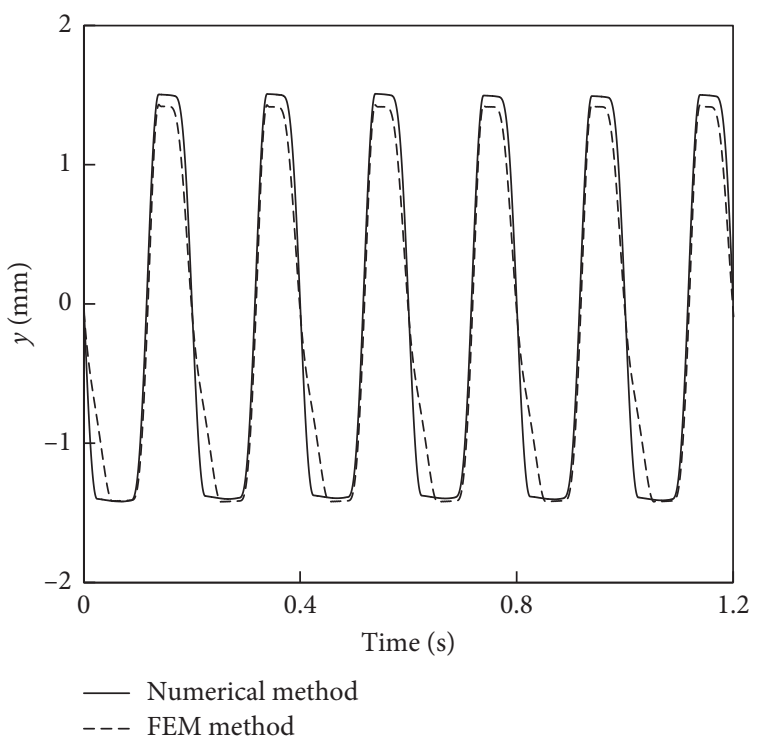

(c)

FIgURE 11: The time-domain curves of displacement of the numerical method and FEM method for different excitation frequencies. (a) $10 \mathrm{~Hz}$. (b) $7 \mathrm{~Hz}$. (c) $5 \mathrm{~Hz}$.

4.2.2. The Effect of Different Friction Coefficients on the Response. Dry friction is the main form of railway freight car damper. The coefficient of dry friction affects the damping performance of the wagon. Simulation is carried out based on the proposed model to describe the relationship between the dry friction and damping. The friction coefficients of interfaces of both wedge-side frame and wedge-bolster are set to the same, and the friction coefficient varies from 0.12 to 0.44 . The displacement response of the suspension system is shown in Figure 16. With the friction coefficient of contact surfaces, the time domain waveform of the relative displacement response has changed, and it appears that the time of a constant displacement during part of a period is increasing. When the system movement shows sticking events, the suspension will be invalid, and it will no longer have the function of vibration absorption, and the high-frequency vibration transmitted to the car body affects the safety of wagon driving. This may be the reason that the relative friction factor of the bogie friction damping device is too large, causing the vertical vibration of the vehicle to deteriorate in the literature [30].

4.2.3. The Effect of Different Side Frame Angles on the Response. The angle of the side frame ranges from -4 to 6 degrees. The displacement response of the suspension system is shown in Figure 17. When the angle of the side frame 


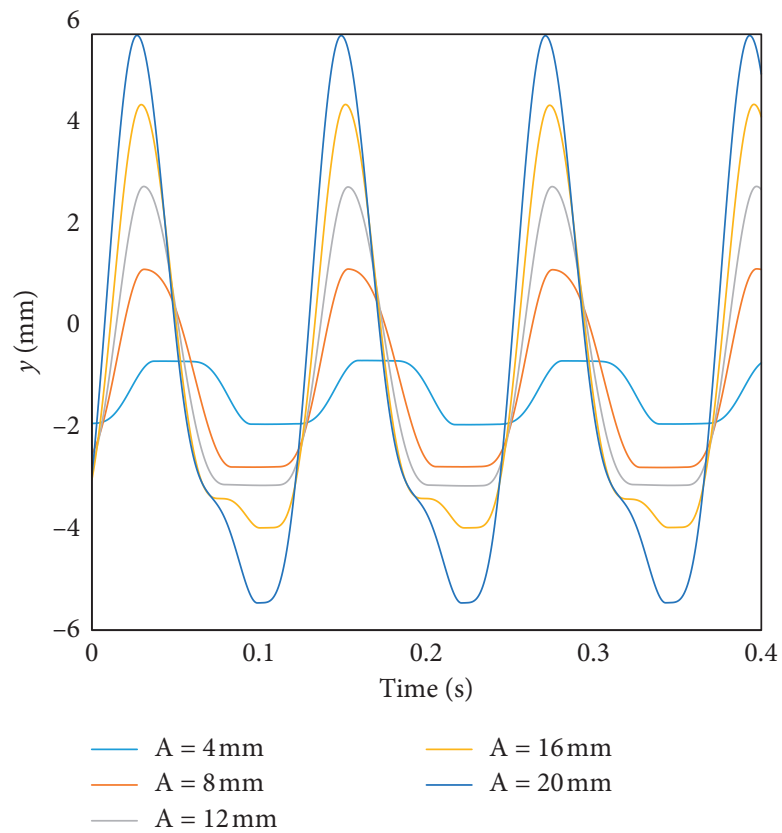

FIGURE 12: The influence of excitation amplitude on the response of the suspension system when excitation frequency $=7 \mathrm{~Hz}$.

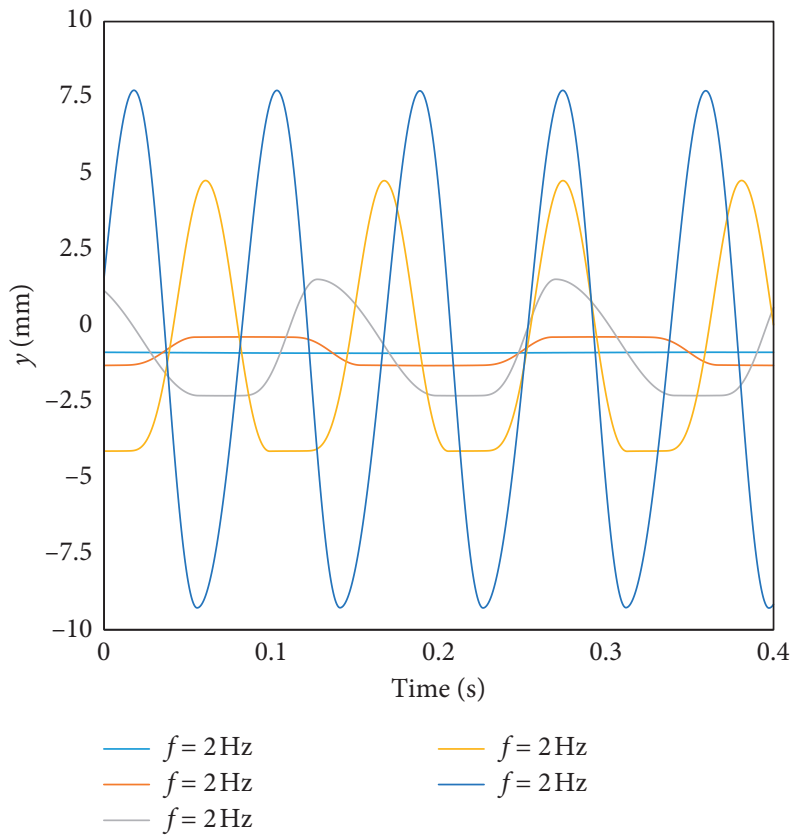

Figure 13: The influence of excitation amplitude on the response of the suspension system when excitation amplitude $=15 \mathrm{~mm}$.

increases, the slope to the trough decreases. When the relative displacement reaches the trough, the length of the stick time remains the same. When the angle of side fame changes from negative to positive and gradually increases, the distance between the two sides of the side frame in the $X$ direction increases as the relative movement in the $Y$ direction decreases. The proportion of the frictional force in the $Y$-direction resultant force decreases as the pressure on the friction surface decreases, and the effect of the vertical spring stiffness will increase. Therefore, when the friction force increases in the $Y$ direction, the acceleration in the $Y$ direction is suppressed from increasing rapidly, and the overall movement is more stable; otherwise, the overall motion is suppressed later, and the overall high-frequency energy is higher than that shown in Figure 11(b).

4.2.4. The Effect of Different Wedge Angles on the Response. The angle of wedge increases from 20 to 70 degrees. The displacement response of the suspension system is shown in Figure 18. When the wedge angle decreases, the sticking time of trough in the displacement response curves increases. 


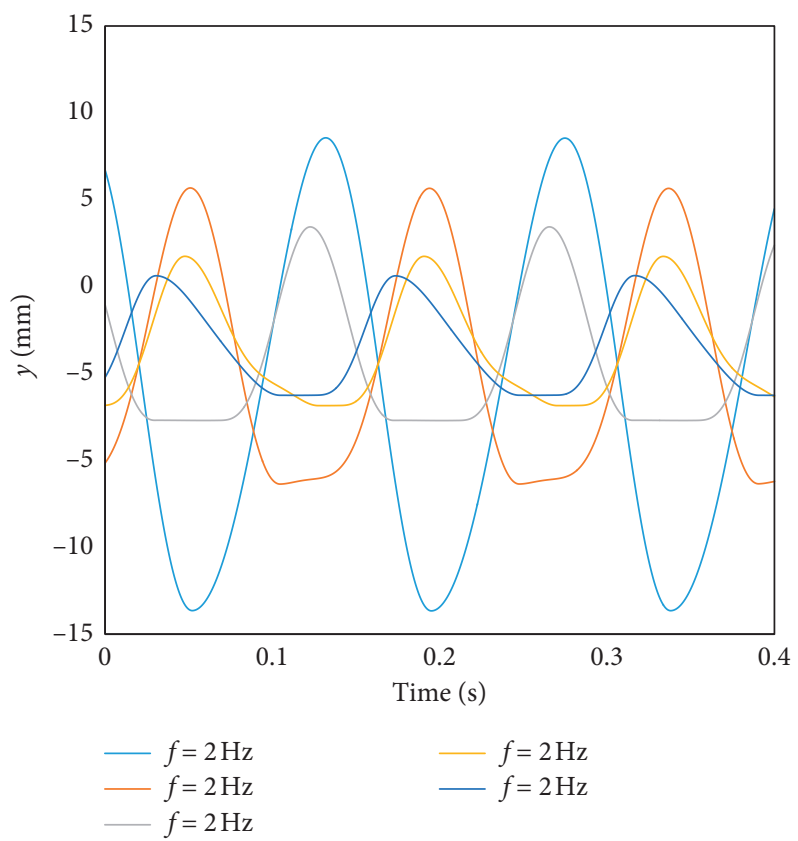

Figure 14: The influence of bolster stiffness on the response of the suspension system when excitation amplitude $=15 \mathrm{~mm}$ and frequency $=7 \mathrm{~Hz}$.

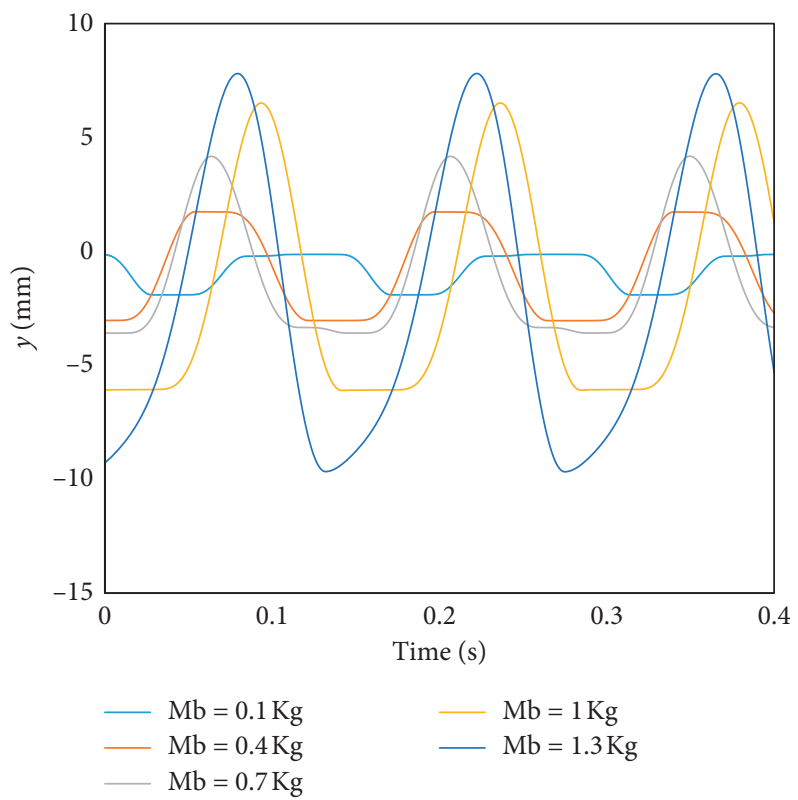

Figure 15: The influence of bolster mass on the response of the suspension system when excitation amplitude $=15 \mathrm{~mm}$ and frequency $=7 \mathrm{~Hz}$. 


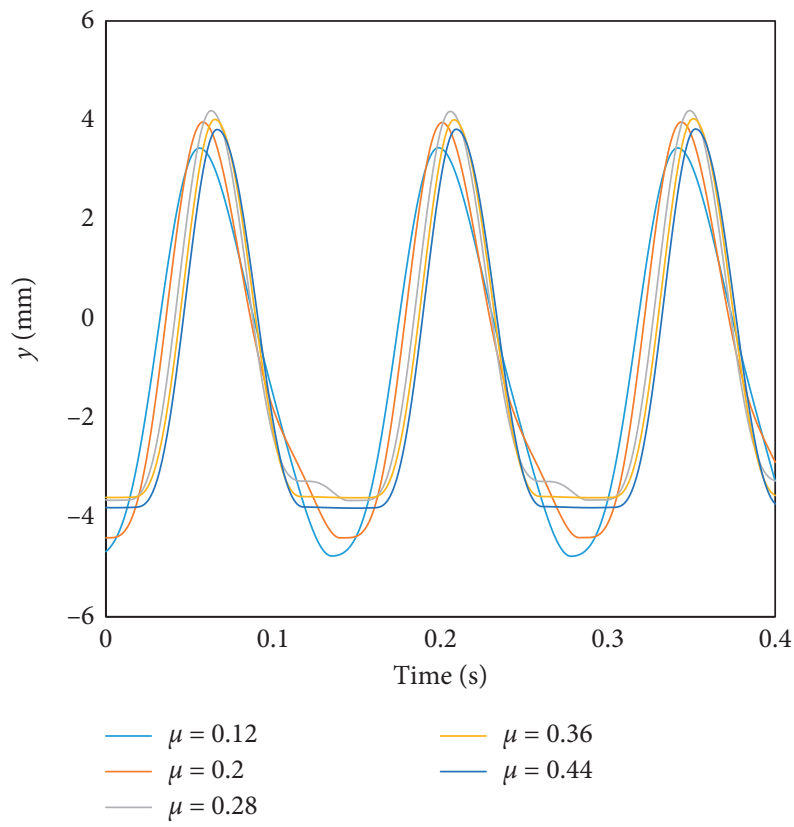

FIGURE 16: The influence of friction coefficient on the response of the suspension system when excitation amplitude $=15 \mathrm{~mm}$ and frequency $=7 \mathrm{~Hz}$.

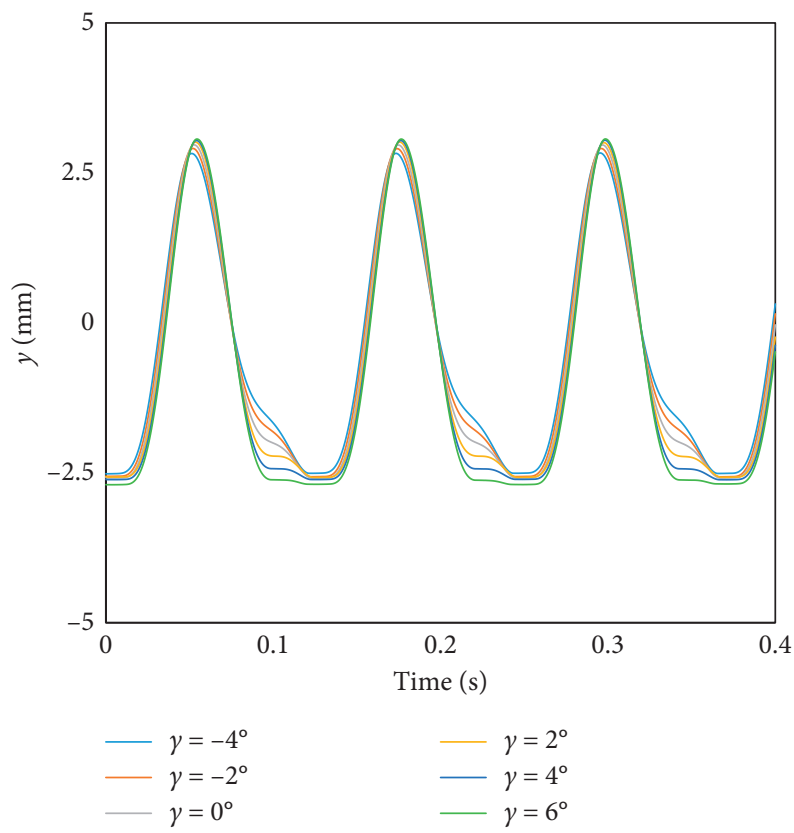

Figure 17: The influence of side frame angle on the response of the suspension system when excitation amplitude $=15 \mathrm{~mm}$ and frequency $=7 \mathrm{~Hz}$. 


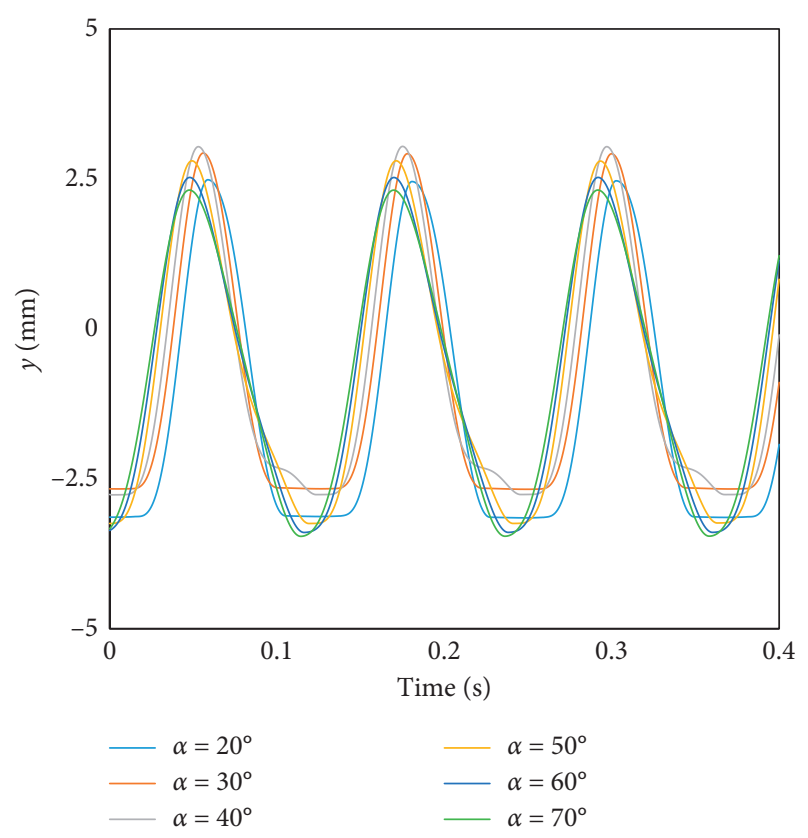

Figure 18: The influence of wedge angle on the response of the suspension system when excitation amplitude $=15 \mathrm{~mm}$ and frequency $=7 \mathrm{~Hz}$.

Increasing the angle of the wedge makes the side pressure decrease, and the friction force decreases with the constant friction coefficient, which can avoid the occurrence of sticking events. The conclusion obtained by increasing the angle of the wedge is basically the same as that of the method of reducing the friction coefficient.

\section{Conclusions}

This paper established a wedge friction damper model to analyze the dynamic response of several critical structure parameters. A model for the vertical response analysis of a simple suspension element is developed, including bolster, side-frame, and wedges. Dry friction sliding contact exists between the bolster and the wedge, as well as between the wedge and the side-frame wall.

The displacement and velocity of bolster relative to side frame are analyzed by analytical method, numerical method, and FEM. The advantages, disadvantages, and application scope of these three methods are discussed. Results show that the numerical method is the most suitable method to analyze the adhesion slip phenomenon and the influence of structural components on the vibration isolation state in a period.

The effects of friction coefficient, geometry, and excitation characteristics on the dynamic response of the secondary suspension are analyzed by numerical method. The sticking phenomenon mostly appears at the maximum and minimum relative displacement of the suspension. The sticking phenomenon is related to the excitation energy of the system, and increasing excitation amplitude or frequency will suppress sticking events. Reduced spring stiffness or system mass of suspension can reduce sticking events. The increase of the angle of the side frame will prolong the sticking time. By increasing the friction coefficient and the angle of the wedge, the sticking events of the system become more obvious. The sticking events will cause the high-frequency energy of the system to increase, making the system's vibration acceleration greater. This phenomenon is consistent with reducing the vertical vibration of the railway vehicle by reducing the spring stiffness of the friction coefficient in the literature [31].

To the best of the authors' knowledge, there is no analysis of the three calculation methods at the same time or verification for the accuracy of each other. The influence of different structural parameters and excitation on the suspension system is analyzed in this paper. However, this paper only considers the vertical motion of the suspension system, which cannot fully reflect the vibration characteristics of the system. But it can also provide guidance for the selection of methods in the design process of railway vehicle bogies and provide a reference for the failure analysis of wedge.

\section{Data Availability}

The model parameters data used to support the findings of this study are included within Table 1 of the article.

\section{Conflicts of Interest}

The authors declare that they have no conflicts of interest.

\section{Acknowledgments}

This work was funded in part by a grant from the project of QiQiHar Railway Rolling Stock Co., Ltd. (contract T20190035) and by a grant from the National Natural Science Foundation of China (grant numbers 11872044); and by a grant from the National Key Research and Development Program of China (grant number 2018YFB0106200).

\section{References}

[1] S. D. Iwnicki, S. Stichel, A. Orlova, and M. Hecht, "Dynamics of railway freight vehicles," Vehicle System Dynamics, vol. 53, no. 7, pp. 995-1033, 2015.

[2] Y. Boronenko, A. Orlova, and E. Rudakova, "Influence of construction schemes and parameters of three-piece freight bogies on wagon stability, ride and curving qualities," Vehicle System Dynamics, vol. 44, no. sup1, pp. 402-414, 2006.

[3] R. A. Ibrahim, "Friction-induced vibration, chatter, squeal, and chaos-Part I: mechanics of contact and friction," Applied Mechanics Reviews, vol. 47, no. 7, pp. 209-226, 1994.

[4] A. H. Nayfeh and D. T. Mook, Nonlinear Oscillations, Wiley Classics, Hoboken, NJ, USA, 1995.

[5] Q. Wu, C. Cole, M. Spiryagin, and Y. Q. Sun, "A review of dynamics modelling of friction wedge suspensions," Vehicle System Dynamics, vol. 52, no. 11, pp. 1389-1415, 2014.

[6] B. F. Feeny and F. C. Moon, "Bifurcation sequences of a Coulomb friction oscillator," Nonlinear Dynamics, vol. 4, no. 1, pp. 25-37, 1993.

[7] K. Popp and P. Stelter, "Stick-Slip vibrations and chaos," Philosophical Transactions of the Royal Society A: 
Mathematical, Physical Engineering Sciences, vol. 332, no. 1624, pp. 89-105, 1990.

[8] A. B. Kaiser, J. P. Cusumano, and J. F. Gardner, "Modeling and dynamics of friction wedge dampers in railroad freight trucks," Vehicle System Dynamics, vol. 38, no. 1, pp. 55-82, 2002.

[9] B. Sperry, C. Sandu, and B. Ballew, "Complex bogie modeling incorporating advanced friction wedge components," in Proceedings of the 2009 Joint Rail Conference, pp. 127-133, Pueblo, CO, USA, March 2009.

[10] L. Hengli, L. Fu, F. Maoha, Y. Xing, and Z. Chaode, "Dynamic modeling and simulation of wedges friction dampe," Journal of Railway Science and Engineering, vol. 12, no. 5, pp. 11911199, 2015.

[11] N. Yinyu, Data-driven Dynamics Modeling and Simulation Research Forrailway Vehicles, Southwest Jiaotong University, Chengdu, China, 2016.

[12] Y. G. Ye, D. C. Shi, P. Krause, and M. Hecht, "A data-driven method for estimating wheel flat length," Vehicle System Dynamics, vol. 247, 2019 in English.

[13] M. Taheri and M. Ahmadian, "Machine learning from computer simulations with applications in rail vehicle dynamics," Vehicle System Dynamics, vol. 54, no. 5, pp. 653-666, 2016.

[14] S. Bruni, J. Vinolas, M. Berg, O. Polach, and S. Stichel, "Modelling of suspension components in a rail vehicle dynamics context," Vehicle System Dynamics, vol. 49, no. 7, pp. 1021-1072, 2011.

[15] F. Xia, "Modelling of a two-dimensional Coulomb friction oscillator," Journal of Sound and Vibration, vol. 265, no. 5, pp. 1063-1074, 2003.

[16] T. Zhang, H. True, and H. Dai, "The lateral dynamics of a nonsmooth railway wheelset model," International Journal of Bifurcation and Chaos, vol. 28, no. 8, Article ID 1850095, 2018.

[17] L. B. Baruffaldi, "On the application of linear complementarity-based contact to study the dynamic behavior of friction dampers of railway vehicles," Journal of the Brazilian Society of Mechanical Sciences \& Engineering, vol. 40, no. 8, p. 372, 2018.

[18] Y. Q. Sun and C. Colin, "Finite element modeling and analysis of friction wedge damping during suspension bounce modes," Journal of Vibration and Acoustics, vol. 131, no. 5, Article ID 054504, 2009.

[19] Y. Q. Sun and C. Cole, "Finite element modeling and analysis of friction-wedge dampers during suspension pitch modes," Journal of Vibration \& Acoustics, vol. 130, p. 021003, 2008.

[20] L. Hongyou, H. Hongtao, Y. Yuebin, and H. Haibin, "Influence of damping system on the dynamics performance of C80 type aluminium alloy gondola cars," Railway Locomotive \& Car, vol. 3, pp. 5-8, 2007.

[21] Z. Yijiao, Analysis of Load Spectrum and Dynamic Stress Response of C80 Car Body Based on Railway Line Test, Beijing Jiaotong University, Beijing, China, 2015.

[22] N. K. Chandiramani, K. Srinivasan, and J. Nagendra, "Experimental study of stick-slip dynamics in a friction wedge damper," Journal of Sound and Vibration, vol. 291, no. 1-2, pp. 1-18, 2006.

[23] S. L. Lau and Y. K. Cheung, "Amplitude incremental variational principle for nonlinear vibration of elastic systems," Journal of Applied Mechanics, vol. 48, no. 4, p. 959, 1981.

[24] J. Niu, Y. Shen, S. Yang, and S. Li, "Higher-order approximate steady-state solutions for strongly nonlinear systems by the improved incremental harmonic balance method," Journal of Vibration and Control, vol. 24, no. 16, pp. 3744-3757, 2018.
[25] Y. Wu, "The harmonic balance method for Yao-Cheng oscillator," Journal of Low Frequency Noise, Vibration and Active Control, vol. 38, no. 3-4, pp. 1716-1718, 2019.

[26] T. Sauer, Numerical Analysis, Pearson Education, London, UK, 2nd edition, 2012.

[27] C. D. Zhu, Engineering Vibration Foundation, Beihang University Press, Beijing, China, 2004.

[28] W. Ting, Countermeasures Container Flat Car Rigid-Flexible Coupling and Vibration Simulation, Dalian Jiaotong University, Dalian, China, 2008.

[29] C. Haitao, H. Hongtao, and W. Duojun, "Analysis vibration acceleration is too large double container train car or vertical," Railway Vehicle, vol. 42, no. 9, pp. 5-8, 2004.

[30] Y. Aiguo, Y. Lianyou, H. Jianmin, and H. Haibin, "Experimental Study of Friction friction damping device supporting bogie cross," Railway Vehicle, vol. 43, no. 8, pp. 14-18, 2005, in chi.

[31] L. Hongyou, "Impact of ZK6 bogie damping system for dynamic behavior of the C80 aluminum gondola," Symposium on Freight Cars, vol. 1, pp. 63-71, 2006. 